\title{
Comparison of Nutrient Density and Nutrient-to-Cost between Cooked and Canned Beans
}

\author{
Michael Zanovec ${ }^{1}$, Carol E. O’Neil ${ }^{*}$, Theresa A. Nicklas ${ }^{2}$ \\ ${ }^{1}$ School of Human Ecology, Louisiana State University Agricultural Center, Baton Rouge, LA, USA; ${ }^{2}$ USDA/ARS Children's Nutri- \\ tion Research Center, Baylor College of Medicine, Houston, TX, USA. \\ E-mail: coneil1@lsu.edu
}

Received January $6^{\text {th }}, 2011$; revised February $9^{\text {th }}, 2011$; accepted March $4^{\text {th }}, 2011$.

\begin{abstract}
Consumption of nutrient rich foods such as beans and peas is recommended because these foods provide key nutrients and relatively little energy. Many consumers are unfamiliar with dried beans or do not have the time to prepare them. The purpose of this study was to compare nutrient density and nutrient-to-cost among dried cooked, canned (liquid and solids), and canned/drained black, garbanzo, kidney, lima, pinto, white beans, and black-eyed peas. Prices were obtained from 60 grocery stores in January 2009. Nutrient content per $100 \mathrm{~g}$ was calculated using the U.S. Department of Agriculture Nutrient Database for Standard Reference, Release 22, and Nutrition Data System for Research (for canned/drained). Nutrient density scores were estimated using the Nutrient Rich Food Index 9.3 (NRF9.3). Nutrient-to-cost ratio (NTCR) was computed as the NRF 9.3 score (per $100 \mathrm{kcal})$ divided by the cost per half-cup servings per package (12) or per can (3.5). Compared to canned beans, dried cooked beans were significantly more energy dense, contained more protein, fiber, iron, potassium and magnesium; and less sodium than canned beans ( $p<0.05$ for all). Canned/drained beans contained more sodium than cooked beans $(p<0.05)$. NRF9.3 scores were 7.3, 2.8, and 4.8 for cooked, canned, and canned/drained beans, respectively. NTCR for cooked, canned, and canned/drained beans was 63.4, 8.9, and 15.2, respectively. Results highlight the benefits of choosing dried beans and also illustrate that canned beans, when drained, provide a healthy alternative. Beans, regardless of type/form, are a nutrient rich food and should be encouraged as part of an overall healthy diet.
\end{abstract}

Keywords: Beans, Legumes, Nutrient Density, Nutrient Cost, Food Composition

\section{Introduction}

The U.S. Department of Agriculture (USDA) MyPyramid Food Guidance System translates the 2005 Dietary Guidelines for Americans (DGA) into a total diet that aims to meet nutrient needs from food sources while limiting overconsumption [1]. Consumption of nutrient rich foods such as fruit, vegetables, whole grains, and legumes is encouraged; whereas foods high in saturated fatty acids (SFA), added sugars, and sodium should be limited [2]. For a 2,000 kilocalorie (kcal) diet, MyPyramid recommends three cups of dry beans and peas (referred to in this manuscript as beans) per week as part of an overall healthy diet [3]. Although the individual nutrient content of species varies [4], beans are excellent sources of fiber and folate, and they are good sources of plant protein, non-heme iron, thiamin, magnesium, phos- phorus, potassium, and copper [5]. Beans are also naturally low in sodium.

As part of an overall healthful diet, consumption of beans has been associated with improved weight management [6] and reduced risk of coronary heart disease [7], type 2 diabetes mellitus [8,9], and certain types of cancer [10]. Despite the health benefits of consuming beans, national survey data suggest that individuals do not consume recommended amounts. In the 1999-2002 National Health and Nutrition Examination Survey (NHANES), only $7.9 \%$ of adults aged $19+$ years of age consumed beans [11] the day of the recall. Low intakes were confirmed in another study of individuals aged 2+ years participating in NHANES 2001-2002 [12] which found that $7.1 \%$ of the population reported consuming beans.

In addition to their health benefits, beans are a versa- 
tile, economical and convenient recipe ingredient. Many species of beans are consumed worldwide; however, MyPyramid lists common beans, including black, pinto, lima, kidney, navy, white, black-eyed peas, and chickpeas [13]. All of these items are readily available precooked in cans as well as in dry packaged form $[5,14]$. Canned beans are a quick substitute for preparing dry packaged beans; although the can includes solids and liquids, called brine, which increases the sodium content and when consumed dilutes the nutrient density compared to uncooked dried beans. However, the sodium content of canned beans can be reduced substantially simply by draining (and rinsing) before cooking [15].

Consumption of dried beans improves diet quality [11] and reduces health risks [4-10], however, many consumers are unfamiliar with dried beans or do not have the time to prepare them. The purpose of this study was to compare nutrient density and nutrient-to-cost of cooked, canned, and canned/drained beans. We hypothesized that cooked beans would cost less per unit than canned beans; further, with the possible exception of excess sodium in canned beans, we expected the nutrient density to be similar between cooked and canned/drained beans. After accounting for differences in cost between cooked and canned beans, we also hypothesized that cooked beans would provide significantly higher nutrient-to-cost ratios (i.e., more nutrients at a lower cost) than both canned and canned/drained beans.

\section{Materials and Methods}

\subsection{Food Price Data}

Food prices (in US dollars) were obtained from 60 full-service grocery stores in the Baton Rouge metropolitan area of Southeast Louisiana during the first two weeks of January 2009. Stores were identified via an online search engine using search terms "supermarkets" and "super stores." The Baton Rouge metropolitan area included nine parishes (counties).

Prices were obtained for dry packaged and canned black, garbanzo, kidney, lima, pinto, white beans, and black-eyed peas. For dry packaged beans, the lowest cost item per pound was selected. For canned beans, the lowest priced item or the most economical (based on price per product size) was recorded. If more than one of the same item was available (e.g., multiple brands), the lowest price per unit option was selected. Retail prices for individual types of dried or canned beans "as purchased" were averaged across all 60 stores.

\subsection{Nutrient Composition}

Nutrient content composition data was obtained using two sources: 1) the USDA National Nutrient Database for Standard Reference, Release 22 (SR22) [16]; and 2) the 2009 Nutrition Data System for Research ([NDSR]; Nutrition Coordinating Center, Minneapolis, MN, 2009). The USDA National Nutrient Database is the primary source of food composition data in the United States. The SR22 contains data on 7,538 food items and up to 143 food components. This database is updated every two years and is the underlying source for the dietary intake interview component of the NHANES called What We Eat in America (WWEIA) [17]. One caveat of the current USDA SR22 database is that the nutrient content of canned beans is only available as solids and liquids. This makes direct nutrient comparisons between cooked beans (from dried) and canned beans impossible since the liquids in canned beans dilutes the nutrient density of the solids. The NDSR is another food and nutrient database widely used to collect 24-hour dietary recall data. Unlike the USDA database, NDSR contains nutrient information for canned beans after draining, which allows for a more comparable comparison to cooked beans and also provides an opportunity to examine differences in nutrient content and nutrient density between canned beans with solids and liquids and canned beans after draining. For products where direct comparisons were available (e.g., cooked SR22 vs. cooked NDSR), results were similar.

Nutrient content per 100 grams were calculated for each type and form of bean in each database. For dried beans, nutrient content was for $100 \mathrm{~g}$ edible portion, after cooking, without sodium or fat added. Nutrient analyses of canned and canned/drained beans were also based on $100 \mathrm{~g}$ edible portion.

The price in US dollars of each item was averaged and divided by 100 to reflect average price per $100 \mathrm{~g}$ of the edible portion (in $\$ / 100$ g). Energy density (ED) was defined as the amount of available energy per unit weight of food (in kcal/100 g). Energy cost was defined as the cost of $100 \mathrm{kcal}$ provided by each item (in $\$ / 100 \mathrm{kcal}$ ). Finally, nutrient cost was based on the nutrient density provided by each item divided by the number of servings contained per package (for dried beans) or per can.

\subsection{Calculation of Nutrient Density Scores}

Nutrient density (ND) scores were calculated using the Nutrient Rich Food Index 9.3 (NRF9.3) [18]. The NRF9.3 is calculated as the sum of the percentage of daily values (\% Daily Value [DV]) for nine nutrients to encourage (NR9) minus the sum of the percentage of maximum recommended values for three nutrients to limit (LIM), with all daily values calculated per $100 \mathrm{kcal}$ and capped at $100 \%$. The algorithm used (based on $100 \mathrm{~g}$ ) was computed as follows:

NRF9.3 $=[(\% \mathrm{DV}$ protein $+\% \mathrm{DV}$ fiber $+\% \mathrm{DV}$ vitamin $\mathrm{A}+\% \mathrm{DV}$ vitamin $\mathrm{C}+\% \mathrm{DV}$ vitamin $\mathrm{E}+\% \mathrm{DV}$ calcium $+\% \mathrm{DV}$ magnesium $+\% \mathrm{DV}$ iron $+\% \mathrm{DV}$ potassium) - 
(\%DV saturated fatty acids + \%DV sodium + \% DV total sugars)] / $100 \mathrm{kcal}$

Nutrients to encourage are either from the Food and Drug Administration (FDA) definition of "healthy foods" [19] or were identified as nutrients of concern by the 2005 DGA [2]. Nutrient density scores were calculated for each type of bean and form (cooked, canned, and canned/drained) found in the SR22 or NDSR databases. For each equation, the \%DV was the percent of the DV contributed for that nutrient. The DV reference standards used on the Nutrition Facts panel and based on the Reference Daily Intakes for a 2,000 kcal diet [19] were used in this analysis.

\subsection{Calculation of Nutrient-to-Cost}

The nutrient-to-cost ratio (NTCR) was calculated as the mean NRF9.3 score per $100 \mathrm{kcal}$ divided by the average price in dollars per number of half-cup servings contained within a one pound package of dried beans or per can. Therefore, for cooked beans, the average cost in dollars was divided by 12, the number of half-cup servings in a one pound package of dried beans. For canned beans, average cost in dollars was divided by 3.5 halfcup servings, the number of servings contained in the cans of beans priced.

\subsection{Statistical Analysis}

All analyses were performed by using the Statistical Package for the Social Sciences version 18.0 (IBM SPSS Inc., Chicago, IL). Descriptive statistics were calculated for price, weight, price per unit weight and per serving, nutrient profile, energy density (kcal/100 g), and for the twelve nutrients included in the NRF9.3. Analysis of variance and univariate comparisons of means were used to compare nutrient content, nutrient density, and nutrient-to-cost between cooked, canned, and canned/drained beans. A Bonferroni adjustment was applied to adjust the means for multiple comparisons. Significance was set at $\mathrm{p}<0.05$.

\section{Results}

\subsection{Food Prices and Weights as Purchased}

The overall mean cost of dried packaged and canned beans “as purchased” was $\$ 1.39$ and $\$ 1.11$, respectively. The average weight of dried beans was standard for all types at $16 \mathrm{oz}$ (453.6 g), whereas the average weight of canned beans was $15.72 \mathrm{oz}$ (445.7 g). Among the eight types of beans purchased, dried lima beans were the most expensive (\$1.59) and canned navy beans were the least expensive (\$0.99) (Table 1).

The average price per number of half-cup servings observed across all stores and all types of beans (dry and canned) was $\$ 0.22$. When analyzed separately, the average price per serving was \$0.12 and \$0.32 for cooked and canned beans, respectively. As shown in Table 1, cooked pinto beans were the least expensive per serving (\$0.103), whereas canned lima beans were the most expensive item per serving (\$0.399).

\subsection{Comparison of Nutrient Content}

Overall, cooked beans from the SR22 and NDSR were similar for all nutrients; therefore, data from the SR22 were used in subsequent analyses of cooked beans. Table 2 shows the nutrient content per 100 grams of each type and form of bean. Overall, cooked beans were more energy dense; contained more protein, carbohydrate, fiber; iron, magnesium, phosphorus, potassium, zinc, copper, thiamin, and folate; and less sodium than canned beans ( $p<0.05$ for all).

When comparing the nutrient content of canned beans to canned/drained beans, canned/drained beans were significantly more energy dense (136 kcal vs. 95 kcal; p < $0.05)$; contained more total carbohydrate (24.4 g vs. 17.4 g; p < 0.05); protein (8.5 g vs. 5.7 g; p < 0.05); fiber (7.6 g vs. 4.9 g; $\mathrm{p}<0.05)$; iron (2.56 mg vs. $1.69 \mathrm{mg}$; $\mathrm{p}<$ 0.05); potassium (407 mg vs. $262 \mathrm{mg}$; p < 0.05); folate (141 mcg vs. 57 mcg; p < 0.001); phosphorus (140 mg vs. $94 \mathrm{mg} ; \mathrm{p}<0.001$ ); magnesium (51 mg vs. $35 \mathrm{mg} ; \mathrm{p}<$ $0.05)$; zinc (1.15 mg vs. $0.75 \mathrm{mg}$; $\mathrm{p}<0.01)$; and copper (0.25 mg vs. $0.17 \mathrm{mg} ; \mathrm{p}<0.01$ ) than canned beans (data not shown).

\subsection{Comparison of Nutrient Density and Nutrient-to-Cost}

Table 3 shows the NRF9.3 scores and NTCR for each type and form of bean. Cooked and canned white beans were the most nutrient dense item, with NRF9.3 scores of 8.6 and 8.3, respectively. Among the other types of cooked beans, navy beans ranked second highest (8.1), followed by pinto beans (7.6); lima beans (7.4), black beans (7.3), kidney beans (7.1); black-eyed peas (6.8), and garbanzo beans (5.2) ranked last. For canned beans, the ranking of NRF9.3 scores from highest to lowest was as follows: white (8.3); black (3.2); pinto (2.4); lima (2.0); kidney (1.8); navy and garbanzo (1.7); and black-eyed peas (1.1). Finally, among canned/drained beans, pinto ranked highest (6.1), followed by white (5.6); lima (5.2); kidney and garbanzo (4.4); navy and black (4.3); and black-eyed peas (3.9) ranked last (Table 3).

A summary of NRF9.3 scores and NTCR between cooked, canned, and canned/drained beans is shown in Table 4. Overall, cooked beans were significantly more nutrient dense than canned or canned/drained beans ( $\mathrm{p}<$ 0.01). Mean NRF 9.3 scores of cooked beans (per 100 kcal) was 7.3 compared to 2.8 and 4.8 for canned and 
Table 1. Average price, weight, and price per unit weight for the eight types of beans used in the analysis.

\begin{tabular}{|c|c|c|c|c|c|c|c|c|c|c|c|c|c|c|c|c|}
\hline \multirow{2}{*}{$\begin{array}{l}\text { Bean type } \\
\text { Bean form }\end{array}$} & \multicolumn{2}{|c|}{ Black } & \multicolumn{2}{|c|}{ Black-eyed Peas } & \multicolumn{2}{|c|}{ Garbanzo } & \multicolumn{2}{|c|}{ Kidney } & \multicolumn{2}{|c|}{ Lima } & \multicolumn{2}{|c|}{ Navy } & \multicolumn{2}{|c|}{ Pinto } & \multicolumn{2}{|c|}{ White } \\
\hline & Cooked & Canned & Cooked & Canned & Cooked & Canned & Cooked & Canned & Cooked & Canned & Cooked & Canned & Cooked & Canned & Cooked & Canned \\
\hline Price (\$) & 1.341 & 1.070 & 1.298 & 1.108 & 1.523 & 1.113 & 1.387 & 1.168 & 1.589 & 1.396 & 1.358 & 0.990 & 1.235 & 1.006 & 1.376 & 1.044 \\
\hline Weight (oz) & 16.00 & 15.38 & 16.00 & 15.44 & 16.00 & 16.90 & 16.00 & 15.96 & 16.00 & 15.19 & 16.00 & 15.67 & 16.00 & 15.84 & 16.00 & 15.38 \\
\hline Weight (g) & 453.6 & 436.0 & 453.6 & 437.7 & 453.6 & 479.1 & 453.6 & 452.5 & 453.6 & 430.6 & 453.6 & 444.2 & 453.6 & 449.1 & 453.6 & 436.0 \\
\hline Price (per 100 g) & 0.296 & 0.245 & 0.286 & 0.253 & 0.336 & 0.232 & 0.306 & 0.258 & 0.350 & 0.324 & 0.299 & 0.223 & 0.272 & 0.224 & 0.303 & 0.239 \\
\hline Price (per 100 kcal) & 0.228 & 0.270 & 0.270 & 0.247 & 0.329 & 0.329 & 0.205 & 0.195 & 0.195 & 0.241 & 0.307 & 0.307 & 0.305 & 0.410 & 0.410 & 0.214 \\
\hline $\begin{array}{l}1 / 2 \text { cup servings } \\
\text { (per package or can) }\end{array}$ & 12.0 & 3.5 & 12.0 & 3.5 & 12.0 & 3.5 & 12.0 & 3.5 & 12.0 & 3.5 & 12.0 & 3.5 & 12.0 & 3.5 & 12.0 & 3.5 \\
\hline Price per serving & 0.112 & 0.306 & 0.108 & 0.317 & 0.127 & 0.318 & 0.116 & 0.334 & 0.132 & 0.399 & 0.113 & 0.283 & 0.103 & 0.287 & 0.115 & 0.298 \\
\hline
\end{tabular}

*Note: Prices were obtained from 60 full-service grocery stores in Southeast Louisiana in January of 2009. Abbreviations: \$, price in US dollars; oz, ounce; g, grams; kcal, kilocalories.

Table 2. Nutrient content per 100 grams of cooked, canned, and canned/drained beans used in this study.

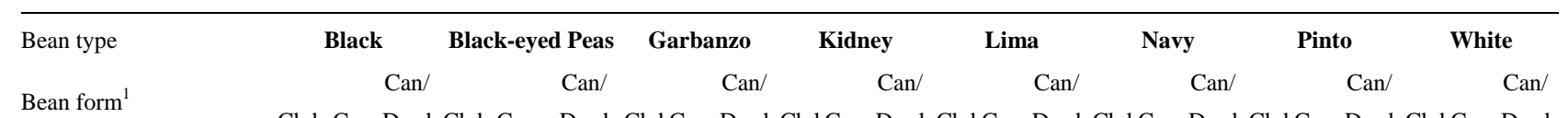

Nutrient

\begin{tabular}{|c|c|c|c|c|c|c|c|c|c|c|c|c|c|c|c|c|c|c|}
\hline Energy (kcal)* & 130 & 91 & 140 & 116 & 77 & 116 & 164119 & 164 & 12784 & 127 & 11579 & 115 & 140113 & 140 & 14386 & 143 & 139114 & 139 \\
\hline rotein $(\mathrm{g})^{*}$ & 8.2 & 6.0 & 8.2 & 7.7 & 4.7 & 7.7 & 8.94 .9 & 8.9 & 8.75 .2 & 8.7 & $\begin{array}{ll}7.8 & 4.9\end{array}$ & 7.8 & 8.27 .5 & 8.2 & $9.0 \quad 4.9$ & 9.0 & $9.7 \quad 7.3$ & 9.7 \\
\hline Total Fat (g) & 0.4 & 0.3 & 0.6 & 0.5 & 0.6 & 0.5 & 2.61 .14 & 2.59 & 0.500 .60 & 0.5 & 0.380 .17 & 0.38 & 0.620 .43 & 0.62 & 0.650 .81 & 0.65 & 0.350 .29 & 0.35 \\
\hline Fiber $(\mathrm{g})^{*, \dagger}$ & 5.3 & 6.9 & 10.5 & 6.5 & 3.3 & 4.53 & $7.6 \quad 4.4$ & 6.19 & 6.45 .3 & 6.4 & $7.0 \quad 4.8$ & 7.0 & 10.55 .1 & 10.5 & $9.0 \quad 4.6$ & 9.0 & 6.34 .8 & 6.3 \\
\hline Total Sugars (g) & 0.3 & 0.4 & 0.4 & 3.3 & 0.4 & 0.4 & 4.80 .4 & 0.4 & 0.31 .9 & 0.9 & 2.90 .7 & 0.7 & $0.4 \quad 0.3$ & 0.4 & 0.30 .2 & 0.3 & 0.30 .3 & 0.5 \\
\hline Calcium (mg) & 55 & 35 & 69 & 24 & 20 & 24 & $49 \quad 32$ & 49 & $35 \quad 34$ & 35 & $17 \quad 21$ & 17 & $69 \quad 47$ & 69 & $46 \quad 43$ & 46 & $90 \quad 73$ & 90 \\
\hline Iron (mg) ${ }^{*, \dagger}$ & 2.9 & 1.9 & 2.4 & 2.5 & 1.0 & 2.5 & 2.91 .4 & 2.9 & 2.21 .2 & 2.2 & 2.41 .8 & 2.4 & 2.41 .9 & 2.4 & 2.11 .5 & 2.1 & 3.73 .0 & 3.7 \\
\hline Magnesium (mg) ${ }^{*, \dagger}$ & 49 & 35 & 53 & 53 & 28 & 53 & $48 \quad 29$ & 48 & $42 \quad 27$ & 42 & $43 \quad 39$ & 43 & $53 \quad 47$ & 53 & $50 \quad 27$ & 50 & $63 \quad 51$ & 63 \\
\hline Phosphorus (mg) ${ }^{*}, \dagger$ & 152 & 108 & 144 & 156 & 70 & 156 & 16890 & 168 & 13890 & 138 & 11174 & 111 & 144134 & 144 & 14792 & 147 & 11391 & 113 \\
\hline Potassium (mg) ${ }^{*, \dagger}$ & 433 & 308 & 389 & 278 & 172 & 278 & 291172 & 291 & 405237 & 405 & 508220 & 508 & 389288 & 389 & 436243 & 436 & 561454 & 561 \\
\hline Sodium (mg)* & 3 & 384 & 385 & 4 & 299 & 240 & 7299 & 141 & 1296 & 237 & 2336 & 221 & $0 \quad 448$ & 385 & 1294 & 159 & 6 & 308 \\
\hline Zinc (mg) ${ }^{*, \dagger}$ & 0.76 & 0.54 & 1.03 & 1.29 & 0.70 & 1.29 & 1.531 .06 & 1.53 & 1.000 .46 & 1.00 & 0.950 .65 & 0.95 & 1.030 .77 & 1.03 & 0.980 .69 & 0.98 & 1.381 .12 & 1.38 \\
\hline Vitamin C (mg) & 0.0 & 2.7 & 0.9 & 0.4 & 2.7 & 0.4 & $1.3 \quad 3.8$ & 1.3 & 1.21 .2 & 1.2 & $0.0 \quad 0.0$ & 0.0 & $\begin{array}{ll}0.9 & 0.7\end{array}$ & 0.9 & $\begin{array}{ll}0.8 & 0.9\end{array}$ & 0.8 & $0.0 \quad 0.0$ & 0.0 \\
\hline Thiamin (mg) ${ }^{*, \dagger}$ & 0.23 & 0.14 & 0.24 & 0.20 & 0.08 & 0.20 & 0.120 .03 & 0.12 & 0.160 .12 & 0.16 & 0.160 .06 & 0.16 & 0.240 .14 & 0.24 & 0.190 .10 & 0.19 & 0.120 .10 & 0.12 \\
\hline iboflavin (mg) & 0.06 & 0.12 & 0.07 & 0.06 & 0.07 & 0.06 & 0.060 .03 & 0.06 & 0.060 .05 & 0.06 & 0.060 .03 & 0.06 & 0.070 .06 & 0.07 & 0.060 .06 & 0.06 & 0.050 .04 & 0.05 \\
\hline Niacin (mg) & 0.53 & 0.62 & 0.65 & 0.50 & 0.35 & 0.50 & 0.530 .14 & 0.53 & 0.580 .41 & 0.58 & 0.420 .26 & 0.42 & 0.650 .49 & 0.65 & 0.320 .29 & 0.32 & 0.140 .11 & 0.14 \\
\hline Pantothenic acid (mg) & 0.26 & 0.18 & 0.27 & 0.41 & 0.19 & 0.41 & 0.290 .30 & 0.29 & 0.220 .14 & 0.22 & 0.420 .26 & 0.42 & 0.270 .17 & 0.27 & 0.210 .14 & 0.21 & 0.230 .19 & 0.23 \\
\hline Vitamin B-6 (mg) & 0.08 & 0.06 & 0.14 & 0.10 & 0.05 & 0.10 & 0.140 .47 & 0.14 & 0.120 .07 & 0.12 & 0.160 .09 & 0.16 & 0.140 .10 & 0.14 & 0.230 .07 & 0.23 & 0.090 .08 & 0.09 \\
\hline Folate (DFE) ${ }^{*, \dagger}$ & 86 & 61 & 140 & 208 & 51 & 208 & 17267 & 172 & $130 \quad 36$ & 130 & $83 \quad 50$ & 83 & 14062 & 140 & 17260 & 172 & $81 \quad 65$ & 81 \\
\hline Vitamin B-12 (mcg) & 0 & 0 & 0 & 0 & 0 & 0 & 0 & 0 & 0 & 0 & 0 & 0 & 0 & 0 & 0 & 0 & 0 & 0 \\
\hline Vitamin A (IU) & 0 & 4 & 0 & 15 & 13 & 15 & $27 \quad 21$ & 27 & 0 & 0 & 0 & 0 & 0 & 0 & 0 & 0 & 0 & 0 \\
\hline Vitamin D (IU) & 0 & 0 & 0 & 0 & 0 & 0 & 0 & 0 & 0 & 0 & 0 & 0 & 0 & 0 & 0 & 0 & 0 & 0 \\
\hline $\mathrm{E}(\mathrm{mg} \mathrm{AT})$ & 0.87 & 0.01 & 0.01 & 0.28 & 0.28 & 0.28 & 0.350 .35 & 0.35 & 0.030 .02 & 0.03 & 0.180 .18 & 0.18 & 0.010 .78 & 0.01 & 0.940 .57 & 0.94 & 0.940 .79 & 0.94 \\
\hline SFA (g) & 0.09 & 0.08 & 0.10 & 0.14 & 0.14 & 0.14 & 0.270 .12 & 0.27 & 0.070 .14 & 0.07 & 0.090 .04 & 0.09 & 0.100 .11 & 0.10 & 0.140 .17 & 0.14 & 0.090 .08 & 0.09 \\
\hline
\end{tabular}


Table 3. Comparison of nutrient density scores ${ }^{1,2}$ and nutrient-to-cost of cooked, canned, and canned/drained beans.

\begin{tabular}{|c|c|c|c|c|c|c|c|c|c|c|c|c|c|c|c|c|}
\hline \multirow{3}{*}{$\begin{array}{l}\text { Bean type } \\
\text { Bean form³ }\end{array}$} & \multicolumn{2}{|c|}{ Black } & \multicolumn{2}{|c|}{ Black-eyed Peas } & \multicolumn{2}{|c|}{ Garbanzo } & \multicolumn{2}{|c|}{ Kidney } & \multicolumn{2}{|c|}{ Lima } & \multicolumn{2}{|c|}{ Navy } & \multicolumn{2}{|c|}{ Pinto } & \multicolumn{2}{|c|}{ White } \\
\hline & & Can/ & & $11 /$ & & $\mathrm{n} /$ & & Can/ & & Can/ & & Can/ & & Can/ & & Can/ \\
\hline & CkdCan & Drnd & CkdCan & Drnd & CkdCan & Drnd & CkdCan & Drnd & CkdCan & Drnd & CkdCan & Drnd & CkdCan & Drnd & CkdCan & Drnd \\
\hline $\begin{array}{c}\text { (kcal/100g) } \\
\text { \%DV }\end{array}$ & 13091 & 140 & 11677 & 116 & 164119 & 164 & 12784 & 127 & 11579 & 115 & 140113 & 140 & 14386 & 143 & 139114 & 139 \\
\hline Protein (g) & 16.412 .1 & 16.5 & 15.59 .5 & 15.5 & 17.79 .9 & 17.7 & 17.310.4 & 17.3 & 15.69 .9 & 15.6 & 16.515 .1 & 16.5 & 18.09 .7 & 18.0 & 19.514 .5 & 19.5 \\
\hline Fiber (g) & 21.227.6 & 42.0 & 26.013 .2 & 18.1 & 30.417 .6 & 24.8 & 25.621 .2 & 25.6 & 28.019 .2 & 28.0 & 42.020 .4 & 42.0 & 36.018 .4 & 36.0 & 25.219 .2 & 25.2 \\
\hline Vitamin A (IU) & $\begin{array}{ll}0.0 & 0.1\end{array}$ & 0.0 & 0.30 .3 & 0.3 & 0.50 .4 & 0.5 & $\begin{array}{ll}0.0 & 0.0\end{array}$ & 0.0 & 0.00 .0 & 0.0 & $0.0 \quad 0.0$ & 0.0 & 0.00 .0 & 0.0 & $\begin{array}{ll}0.0 & 0.0\end{array}$ & 0.0 \\
\hline Vitamin C (mg) & 0.04 .5 & 1.5 & 0.74 .5 & 0.7 & 2.26 .3 & 2.2 & 2.02 .0 & 2.0 & 0.00 .0 & 0.0 & 1.51 .2 & 1.5 & 1.31 .5 & 1.3 & 0.00 .0 & 0.0 \\
\hline Vitamin E (mg AT) & $4.4 \quad 0.1$ & 0.1 & 1.41 .4 & 1.4 & 1.81 .8 & 1.8 & 0.20 .1 & 0.2 & $\begin{array}{lll}0.9 & 0.9\end{array}$ & 0.9 & 0.13 .9 & 0.1 & 4.72 .9 & 4.7 & 4.74 .0 & 4.7 \\
\hline Calcium (mg) & 5.53 .5 & 6.9 & 2.42 .0 & 2.4 & 4.93 .2 & 4.9 & 3.53 .4 & 3.5 & 1.72 .1 & 1.7 & 6.94 .7 & 6.9 & 4.64 .3 & 4.6 & 9.07 .3 & 9.0 \\
\hline Iron (mg) & 15.810 .6 & 13.1 & 13.95 .4 & 13.9 & 16.17 .5 & 16.1 & 12.36 .5 & 12.3 & 13.310 .1 & 13.3 & 13.110.3 & 13.1 & 11.68 .1 & 11.6 & 20.616 .6 & 20.6 \\
\hline Potassium (mg) & 12.48 .8 & 11.1 & 7.94 .9 & 7.9 & 8.34 .9 & 8.3 & 11.66 .8 & 11.6 & 14.56 .3 & 14.5 & 11.18 .2 & 11.1 & 12.56 .9 & 12.5 & 16.013 .0 & 16.0 \\
\hline Magnesium (mg) & 12.38 .8 & 13.3 & 13.37 .0 & 13.3 & 12.07 .3 & 12.0 & 10.56 .8 & 10.5 & 10.89 .8 & 10.8 & 13.311 .8 & 13.3 & 12.56 .8 & 12.5 & 15.812 .8 & 15.8 \\
\hline NR9 & 7.519.27 & 8.28 & 7.796 .95 & 7.04 & 6.365 .50 & 5.98 & 7.267.56 & 7.26 & 8.198.18 & 8.19 & 8.287.42 & 8.28 & 7.867 .57 & 7.86 & 8.858.51 & 8.85 \\
\hline SFA (g) & 0.40 .4 & 0.5 & $\begin{array}{ll}0.7 & 0.7\end{array}$ & 0.7 & 1.30 .6 & 1.3 & 0.40 .7 & 0.4 & $\begin{array}{ll}0.4 & 0.2\end{array}$ & 0.4 & 0.50 .6 & 0.5 & 0.70 .8 & 0.7 & 0.50 .4 & 0.5 \\
\hline Total sugars (g) & 0.30 .3 & 0.3 & 2.60 .3 & 0.3 & 3.80 .4 & 0.4 & 0.31 .5 & 0.7 & 2.30 .6 & 0.6 & 0.30 .2 & 0.3 & 0.30 .2 & 0.3 & 0.30 .2 & 0.4 \\
\hline Sodium (mg) & 0.116 .0 & 16.0 & 0.212 .5 & 10.0 & 0.312 .5 & 5.9 & 0.012 .3 & 9.9 & 0.114 .0 & 9.2 & 0.018 .7 & 16.0 & 0.012 .3 & 6.6 & 0.30 .2 & 12.8 \\
\hline LIM & 0.216 .11 & 4.01 & 1.005 .85 & 3.17 & 1.113 .75 & 1.54 & 0.175 .76 & 2.88 & 0.836 .23 & 2.96 & 0.195 .74 & 4.01 & 0.235 .14 & 1.77 & 0.230 .24 & 3.28 \\
\hline $\begin{array}{c}\text { NRF9.3 } \\
\text { (per 100g) }\end{array}$ & 9.52 .9 & 6.0 & 7.90 .8 & 4.5 & 8.62 .1 & 7.3 & 9.01 .5 & 5.6 & 8.51 .5 & 6.0 & 11.31 .9 & 6.0 & 10.92 .1 & 8.7 & 12.09 .4 & 7.7 \\
\hline $\begin{array}{c}\text { NRF9.3 } \\
\text { (per 100kcal) }\end{array}$ & 7.33 .2 & 4.3 & 6.81 .1 & 3.9 & 5.21 .7 & 4.4 & 7.11 .8 & 4.4 & 7.42 .0 & 5.2 & 8.11 .7 & 4.3 & 7.62 .4 & 6.1 & 8.68 .3 & 5.6 \\
\hline $\mathrm{NTCR}^{4}$ & 65.310 .3 & 14.0 & 62.83 .5 & 12.2 & 41.35 .5 & 14.0 & 61.35 .4 & 13.1 & 55.64 .9 & 13.1 & 71.66 .0 & 15.1 & 74.28 .5 & 21.2 & 75.127.7 & 18.7 \\
\hline
\end{tabular}

${ }^{1}$ NRF scores were calculated as the sum of the DV of nutrients to encourage and subtracting the DV for LIM: NRF9.3 = (protein g/50 g + fiber g/25 g + vitamin A IU/5000 IU + vitamin C mg/60 mg + vitamin E IU/30 IU + calcium mg/1000 mg + iron mg/18 mg + magnesium mg/400 mg + potassium mg/3500 mg saturated fat g/20 g - total sugars g/125 g - sodium mg/2400 mg) $\times 100 \mathrm{kcal} .{ }^{2}$ Indices were calculated per $100 \mathrm{~g}$ and per $100 \mathrm{kcal} .{ }^{3} \mathrm{Ckd}=\mathrm{cooked}$; Can $=$ canned; Can/Drnd = canned/drained. ${ }^{4}$ Nutrient-to-cost ratios (NTCR) were calculated as follows: NRF9.3 Means per 100 kcal / price in US dollars per number of cooked servings per package/can. Abbreviations: \%DV, percent daily value; NR9, sum of percent daily values of nine nutrients to encourage; LIM, sum of percent daily values of three nutrients to limit; NRF9.3, nutrient rich food index 9.3.

Table 4. Summary of NRF9.3 components and nutrient-to-cost between cooked, canned, and canned/drained beans.

\begin{tabular}{cccccccccc}
\hline \%DV & \multicolumn{3}{c}{ Cooked } & \multicolumn{3}{c}{ Canned } & \multicolumn{4}{c}{ Canned/Drained } \\
& M & \pm & SD & M & \pm & SD & M & \pm & SD \\
\hline Energy density (kcal/100 g) & 6.71 & \pm & $0.80^{\ddagger}$ & 4.77 & \pm & $0.86^{*}$ & 6.78 & \pm & 0.8 \\
Protein (g) & 17.05 & \pm & $1.35^{\ddagger}$ & 11.38 & \pm & $2.26^{*}$ & 17.07 & \pm & 1.34 \\
Fiber (g) & 29.3 & \pm & $6.72^{\dagger}$ & 19.6 & \pm & $4.03^{*}$ & 30.21 & \pm & 8.77 \\
Vitamin A (IU) & 0.11 & \pm & 0.21 & 0.1 & \pm & 0.16 & 0.11 & \pm & 0.21 \\
Vitamin C (mg) & 0.96 & \pm & 0.91 & 2.5 & \pm & 2.34 & 1.15 & \pm & 0.84 \\
Vitamin E (mg AT) & 2.25 & \pm & 2.02 & 1.86 & \pm & 1.56 & 1.71 & \pm & 1.95 \\
Calcium (mg) & 4.81 & \pm & 2.38 & 3.81 & \pm & 1.69 & 4.99 & \pm & 2.49 \\
Iron (mg) & 14.59 & \pm & $2.87^{\dagger}$ & 9.38 & \pm & $3.47^{*}$ & 14.25 & \pm & 2.86 \\
Potassium (mg) & 11.79 & \pm & $2.77^{\dagger}$ & 7.48 & \pm & $2.62^{*}$ & 11.63 & \pm & 2.77 \\
Magnesium (mg) & 12.53 & \pm & $1.65^{\ddagger}$ & 8.84 & \pm & $2.37 *$ & 12.66 & \pm & 1.66 \\
NR9 & 7.76 & \pm & 0.75 & 7.62 & \pm & 1.12 & 7.72 & \pm & 0.91 \\
Saturated fat (g) & 0.62 & \pm & 0.32 & 0.55 & \pm & 0.21 & 0.62 & \pm & 0.31 \\
Total sugar (g) & 1.27 & \pm & 1.44 & 0.46 & \pm & 0.43 & 0.41 & \pm & 0.16 \\
Sodium (mg) & 0.13 & \pm & $0.11^{\ddagger}$ & 12.3 & \pm & 5.39 & 10.81 & \pm & 3.87 \\
LIM & 0.5 & \pm & $0.41^{\ddagger}$ & 4.85 & \pm & $2.02^{*}$ & 2.95 & \pm & 0.91 \\
NRF9.3 Mean Score & 7.27 & \pm & $1.0^{\ddagger}$ & 2.77 & \pm & $2.30^{*}$ & 4.77 & \pm & 0.77 \\
Nutrient-to-cost ratio & 63.39 & \pm & $11.20^{\ddagger}$ & 8.96 & \pm & 7.88 & 15.17 & \pm & 3.13 \\
\hline
\end{tabular}

${ }^{\dagger} \mathrm{p}<0.05$, cooked vs. canned; ${ }^{\ddagger} \mathrm{p}<0.01$, cooked vs. canned; ${ }^{*} \mathrm{p}<0.05$, canned vs. canned/drained Abbreviations: \%DV, percent daily value; NR9, sum of percent daily values of nine nutrients to encourage; LIM, sum of percent daily values of three nutrients to limit; NRF9.3, nutrient rich food index 9.3. 
canned/drained beans, respectively (Table 4). Similarly, cooked beans had significantly higher NTCR (63.4 vs. 9.0, and 15.2; $\mathrm{p}<0.01$ ) compared to canned and canned/ drained beans, which indicated that cooked beans provided essential nutrients at a lower cost.

\section{Discussion}

This study found that beans, regardless of type or form, are a nutrient rich food source that contributes substantial amounts of key nutrients in the diet. Beans are produced and widely consumed throughout the world, particularly in Africa, Asia, and Latin America. For people who live in those areas, beans constitute an important source of plant protein, fiber, vitamins and minerals. Beans are high in protein; however, unlike meat, protein from beans does not provide all of the essential amino acids [20]. However, when consumed with whole grains such as brown rice, beans provide a virtually fat-free complete protein at a lesser cost than meat [5]. Although they are widely consumed throughout the world, beans may not be fully appreciated by American consumers, as evidenced by their low consumption [11,12].

With increasing demands placed on families and decreasing amounts of time spent on food preparation, there is a tradeoff between cost and convenience when selecting foods [21]. Food choices are largely driven by taste, cost, and convenience [22]. Barriers to meeting dietary guidelines, particularly among low-income households, include cost [23,24], convenience [22], taste [22], availability [25,26], quality [27], and time [28]. Unlike canned beans which are cooked when purchased, dried beans require a significant amount of preparation time. Dried beans are usually soaked and then cooked for an additional1-2 hours [5]. Most dried beans will triple in volume when soaked or cooked, so that 1-cup of dry packaged beans equals 3 cups cooked.

Data from the 2003-2004 American Time Use Survey [28] found that women, regardless of income and marital status, spend more time cooking than men. Among women, the average time spent cooking ranged from approximately 40 minutes per day for full-time working women to just over 70 minutes per day for nonworking women. Moreover, the total time available for food preparation was dependent upon the number of adults and children in the household and the number of hours spent working. Thus, preparation time is a major consideration. Canned bean products account for approximately $60 \%$ of total bean consumption in the U.S. due primarily to their convenience, ease of preparation, and practicality $[5,14]$.

The concept of nutrient density is an important topic, and several scoring methodologies have been proposed, all with the intent to maximize the amount of essential or shortfall nutrients while minimizing certain nutrients to limit. Rarely do these methods take into account food preferences, food prices, or diet costs. The NRF9.3 method has been validated against the Healthy Eating Index-2005 [29], a measure of diet quality, using 19992002 NHANES dietary intake data collected on participants aged 4 years and older [18]. In addition, Drewnowski [30] recently showed that the NRF9.3 index can help identify healthy, affordable foods. In fact, beans, nuts and seeds were among the most nutrient dense and the most economical, with an average NRF9.3 score or 23.1 per $100 \mathrm{kcal}$ and 282 per U.S. dollar.

In terms of nutrient composition, this study found that the nutrient density score of cooked beans was significantly higher compared to canned beans and canned/ drained beans. This difference can likely be attributed to differences in the sodium content of canned beans, even after draining. High sodium intake is associated with a variety of health conditions, notably high blood pressure and stroke [31]. The average sodium content of canned beans in this study was $295 \mathrm{mg}$ (12.3\% DV). According to recent data from NHANES 2003-2006, usual intake of sodium was approximately 3,400 mg per day [32]. The maximum recommended level issued by the Food and Drug Administration (FDA) is currently 2,400 mg/day for a 2,000 kcal diet [18]. The 2010 Dietary Guidelines Advisory Committee (DGAC) recently recommended that sodium intake should be limited to 1,500 milligrams per day [33], which is consistent with the Institute of Medicine's (IOM) Dietary Reference Intakes for adults [31]. The DGAC acknowledged that an immediate change of this magnitude would be difficult, given product standards and consumer taste preferences. Therefore, they referred to the recent IOM report, "Strategies to Reduce Sodium Intake in the United States," for a "roadmap" on how to achieve gradual reductions at the population level [34]. An example of how to reduce sodium intake by 45 to $50 \%$ would be to drain and rinse canned beans [15] Nutrition educators can use this information to inform their clients about the importance of draining and rinsing canned beans in order to reduce the sodium content and increase the nutrient density.

The FDA has identified "healthy" foods as those that contain at least $10 \% \mathrm{DV}$ per reference amount of one or more of six nutrients: protein, fiber, vitamins A, C, calcium, or iron [19] while being low in total fat, SFA, cholesterol, and sodium. Therefore, foods are disqualified from health claims if a serving of food contains $>13 \mathrm{~g}$ of fat, $>4 \mathrm{~g}$ of SFA, $>60 \mathrm{mg}$ of cholesterol, or $>480 \mathrm{mg}$ of sodium [19]. Based on the FDA reference amount customarily consumed, a serving of cooked and canned beans is $90 \mathrm{~g}$ and $130 \mathrm{~g}$, respectively [35]. In this study, a serving of beans provided an average of $<2 \%$ of the DV 
for total fat, $<1 \%$ SFA, $0 \%$ cholesterol; $17 \%$ protein; 8.5\% total carbohydrate, $30.4 \%$ fiber; and $0.1 \%$ sodium (cooked), 16\% (canned), and 14\% (canned/drained). Additionally, a serving of beans provided an average of $31 \%$ DV for folate, $15 \%$ iron, $13 \%$ magnesium, $14 \%$ phosphorus, $12 \%$ potassium, $8 \%$ zinc, $13 \%$ copper, $11 \%$ thiamin, and 8\% vitamin B-6.

Nutrient profiling can be a valuable tool for both nutrition education and dietary guidance. Consumers can use this information to select foods that provide the most nutrients at the lowest cost. In this study, the Nutrient Rich Food Index was used to quantify nutrient density and nutrient cost. This study found that cooked beans provided significantly more nutrients per $100 \mathrm{kcal}$ and per serving. Therefore, cooked beans provide essential nutrients at a more reasonable cost than canned beans and should be encouraged by health professionals. Health professionals should also help individuals learn to prepare dried beans. Canned beans, however, were also nutrient rich and should be encouraged when individuals cannot prepare dried beans.

This study had several limitations. First, prices were obtained during a one month period from stores located within a metropolitan city in the South. Therefore, while these particular food prices are unlikely to change seasonally, the results cannot be generalized to other geographical regions. Another limitation of this study was the choice of nutrient density scoring methodology. The NRF9.3 has been shown to distinguish differences in nutrient density across a wide range of foods from each of the food groups. However, this particular method, unlike other scoring methods, does not include some key nutrients in beans, such as folate, thiamin, iron, phosphorus, zinc, and copper. The beans included in this study provided an average of $31 \%$ of the daily value for folate. Additional nutrient density scoring methods were tested and all yielded similar results.

Overall, cooked beans were significantly more nutrient dense and provided essential nutrients at a more reasonable cost than canned beans. However, these results also illustrate that canned beans, when drained, can provide a more nutrient dense healthy alternative to canned beans with brine. In conclusion, beans, regardless of type or form, are a nutrient rich food that contributes substantial amounts of key nutrients in the diet. Nutrition educators should encourage consumption of beans as part of an overall healthy diet.

\section{Acknowledgements}

The authors would like to sincerely thank Jeff Gillespie, Robert Boucher, Patrick Hatzenbuehler, and Caitlyn Haines for their assistance in collecting food prices information for this study.

\section{REFERENCES}

[1] P. Britten, K. Marcoe, S. Yamini and C. Davis, "Development of Food Intake Patterns for the MyPyramid Food Guidance System,” Journal of Nutrition Education and Behavior, Vol. 38, No. 6S, 2006, pp. S78-92.

[2] U. S. Department of Agriculture and U.S. Department of Health and Human Services, "Dietary Guidelines for Americans, 2005,” 6th Edition, U. S. Government Printing Office, Washington, DC, 2005.

[3] U. S. Department of Agriculture, "MyPyramid [Web Page]. Inside the Pyramid/Meat \& Beans Group/ How much is needed?” Internet Available: http://www.mypyramid.gov/pyramid/meat_amount_print. aspx [Accessed July 9, 2009].

[4] M. J. Messina, "Legumes and Soybeans: Overview of Their Nutritional Profiles and Health Effects," American Journal of Clinical Nutrition, Vol. 70, 1999, pp. 439S$450 \mathrm{~S}$.

[5] P. B. Geil and J. W. Anderson, "Nutrition and Health Implications of Dry Beans: A Review,” Journal of the American College of Nutrition, Vol. 13, No. 6, 1994, pp. 549-558.

[6] Y. Papanikolaou and V. L. Fulgoni, III, "Bean Consumption is Associated with Greater Nutrient Intake, Reduced Systolic Blood Pressure, Lower Body Weight, and a Smaller Waist Circumference in Adults: Results from the National Health and Nutrition Examination Survey 1999-2002," Journal of the American College of Nutrition, Vol. 27, 2008, pp. 569-576.

[7] L. A. Bazzano, J. He, L. G. Ogden, C. Loria, S. Vupputuri, L. Myers and P. K. Whelton, "Legume Consumption and Risk of Coronary Heart Disease in US Men and Women: NHANES I Epidemiologic Follow-up Study," Archives of Internal Medicine, Vol. 161, No. 21, 2001, pp. 25732578.

[8] B. J. Venn and J. I. Mann, "Cereal Grains, Legumes and Diabetes,” European Journal of Clinical Nutrition, Vol. 58, 2004, pp. 1443-1461.

[9] R. Villegas, Y.-T. Gao, G. Yang, H.-L. Li, T. A. Elasy, W. Zheng and X. O. Shu, "Legume and Soy Food Intake and the Incidence of Type 2 Diabetes in the Shanghai Women's Health Study," American Journal of Clinical Nutrition, Vol. 87, No. 1, 2008, pp. 162-167.

[10] E. Lanza, T. J. Hartman, P. S. Albert, R. Shields, M. Slattery, B. Caan, E. Paskett, F. Iber, J. W. Kikendall, P. Lance, C. Daston and A. Schatzkin, "High Dry Bean Intake and Reduced Risk of Advanced Colorectal Adenoma Recurrence among Participants in the Polyp Prevention Trial,” Journal of Nutrition, Vol. 136, 2006, pp. 1896-1903.

[11] D. C. Mitchell, F. R. Lawrence, T. J. Hartman, J. M. Curran, "Consumption of Dry Beans, Peas, and Lentils Could Improve Diet Quality in the US Population," Journal of the American Dietetic Assocition, Vol. 109, 
No. 5, 2009, pp. 909-913.

[12] J. L. Bachman, J. Reedy, A. F. Subar and S. M. KrebsSmith, "Sources of Food Group Intakes among the US Population, 2001-2002,” Journal of the American Dietetic Assocition, Vol. 108, No. 5, 2008, pp. 804-814.

[13] U. S. Department of Agriculture, "Inside the Pyramid: Dry beans and Peas in the Food Guide," Internet Available:

http://www.mypyramid.gov/pyramid/dry_beans_peas_tab le.html [Accessed April 14, 2009].

[14] G. Lucier, B.-H. Lin, J. Allhouse and L. S. Kantor, "Factors Affecting Dry Bean Consumption in the United States," U.S. Department of Agriculture, Economic Research Service. Vegetables and Specialties Situation \& Outlook Report [VGS-280], Internet Avialable:

http://www.ers.usda.gov/Briefing/DryBeans/PDFs/DryBe anConsumption.pdf [Accessed June 19, 2009].

[15] J. B. Jones and J. R. Mount, "Reducing Sodium Levels in Canned Beans by Draining and Rinsing [abstract]," Presented at: Institute of Food Technologists 2009 annual meeting \& food expo, 2009, Anaheim, CA.

[16] U. S. Department of Agriculture, "Composition of Foods Raw, Processed, Prepared. Usda National Nutrient Database for Standard Reference, Release 22,” 2009.

Internet Available: http://www.ars.usda.gov/nutrientdata [Accessed March 8, 2010].

[17] U. S. Department of Agriculture, Agricultural Research Service, "What We Eat in America," Internet Available: http://www.ars.usda.gov/Services/docs.htm?docid=13793 [Accessed June 8, 2010].

[18] V. L. Fulgoni, D. R. Keast, and A. Drewnowski, "Development and Validation of the Nutrient-Rich Foods Index: A Tool to Measure Nutritional Quality of Foods,” Journal of Nutrition, Vol. 139, No. 8, 2009, pp. 1549-1554.

[19] U. S. Food and Drug Administration. Code of Federal Regulations. Sec 101.9, Nutrition Labeling of Food, 2010. Internet Available:

http://ecfr.gpoaccess.gov/cgi/t/text/text-idx?c=ecfr\&sid= 563f0b6235da3f4c7912a64cbceec305\&rgn=div8\&view= text\&node=21:2.0.1.1.2.1.1.6\&idno=21 [Accessed July 4, 2010].

[20] S. K. Sathe, "Dry Bean Protein Functionality," Critical Reviews in Biotechnology, Vol. 22, No. 2, 2002, pp. 175223.

[21] A. Drewnowski and N. Darmon, "Food Choices and Diet Costs: An Economic Analysis,” Journal of Nutrition, Vol. 135, No. 4, 2005, pp. 900-904.

[22] K. Glanz, M. Basil, E. Maibach, J. Goldberg and D. Snyder, "Why Americans Eat What They do: Taste, Nutrition, Cost, Convenience, and Weight Control Concerns as Influences on Food Consumption,” Journal of the American Dietetic Association, Vol. 98, No, 10, 1998, pp. 11181126.

[23] A. Drewnowski and S. E. Specter, "Poverty and Obesity: The Role of Energy Density and Energy Costs," American Journal of Clinical Nutrition, Vol. 79, No. 1, 2004, pp. 6-16.
[24] D. Cassady, K. M. Jetter and J. Culp, "Is Price a Barrier to Eating More Fruits and Vegetables for Low-Income Families?” Journal of the American Dietetic Associtation, Vol. 107, No. 11, 2007, pp. 1909-1915.

[25] M. S. Amarra, Y. B. Yee and A. Drewnowski, "Symposium on Understanding and Influencing Consumer Food Behaviours for Health: Executive Summary Report," Asia Pacific Journal of Clinical Nutrition, Vol. 17, 2008, pp. 530-539.

[26] A. Cheadle, B. M. Psaty, S. Curry, E. Wagner, P. Diehr, T. Koepsell and A. Kristal, "Community-level comparisons between the grocery store environment and individual dietary practices," Preventive Medicine, Vol. 20, No. 2, 1991, pp. 250-261.

[27] T. Furst, M. Connors, C. A. Bisogni, J. Sobal and L. W. Falk, "Food Choice: A Conceptual Model of the Process," Appetite, Vol. 26, No. 3, 1996, pp. 247-265.

[28] L. Mancino and C. Newman, "Who has Time to Cook? How Family Resources Influence Food Preparation,” U.S. Department of Agriculture, Economic Research Service, Economic Research Report Number 40, 2007.

[29] P. M. Guenther, J. Reedy, S. M. Krebs-Smith, B. B. Reeve and P. P. Basiotis, "Development and Evaluation of the Healthy Eating Index-2005: Technical Report,” Internet Available: http://www.cnpp.usda.gov/HealthyEatingIndex.htm

[30] A. Drewnowski, "The Nutrient Rich Foods Index Helps to Identify Healthy, Affordable Foods," American Journal of Clinical Nutrition, Vol. 91, No. 4, 2010, pp. 1091S1095S.

[31] Institute of Medicine, Food and Nutrition Board, "Dietary Reference Intakes for Water, Potassium, Sodium, Chloride, and Sulfate" Washington, DC, The National Academies Press, 2005.

[32] D. R. Keast and V. L. Fulgoni III, "Dietary Food Sources of Potassium and the Sodium-Potassium Ratio of Foods Consumed in the U.S.: NHANES, 2003-2006” FASEB Journal, Vol. 24, 2010, p. 745.

[33] U.S. Department of Agriculture, Center for Nutrition Policy and Promotion, "Report of the Dietary Guidelines Advisory Committee on the Dietary Guidelines for Americans, 2010," Internet Available:

http://www.cnpp.usda.gov/DGAs2010-DGACReport.htm [Accessed July 2, 2010].

[34] J. E. Henney, C. L. Taylor and C. S. Boon, "Strategies to Reduce Sodium Intake in the United States," 1st Edition. Washington, DC, The National Academies Press, 2010, p. 480 .

[35] U. S. Food and Drug Administration, Electronic Code of Federal Regulations, "Reference Amounts Customarily Consumed Per Eating Occasion [21CFR10112],” Internet Available: http://www.accessdata.fda.gov/scripts/cdrh/cfdocs/c fcfr/CFRSearch.cfm

Page Last Updated: April 1, 2009 [Accessed May 15, 2010]. 